\title{
THE EXISTENCE OF NON-EUCLIDEAN CERCLES DE REMPLISSAGE IN CERTAIN SUBSETS OF THE UNIT DISC
}

\author{
L. H. LANGE
}

The concept of a "set of $\rho$-discs" for complex-valued functions defined in the open unit disc $U$ was introduced in $[4 ; 5$, p. 265] and various results concerning the existence of such sets associated with spirals and Stolz angles were derived. These sets are non-Euclidean analogues to the classical cercles de remplissage of Milloux $[7 ; 8]$. In the present paper additional theorems about Stolz angle phenomena and the existence of $\rho$-discs in more general subsets of $U$ are established and new proofs are indicated for some results of Seidel [9, Theorem 2 and Corollary 1$]$.

It is necessary to recall some definitions. We view the open unit disc $U$ as a non-Euclidean plane of the Poincaré variety, employing the non-Euclidean metric defined by $d\left(z_{0}, z\right)=\frac{1}{2} \log \frac{1+u}{1-u}$, where $u=\left|\frac{z_{0}-z}{1-\bar{z}_{0} z}\right|$, and $z_{0}, z \in U$, [2]. Given a point $\tau$ for which $|\tau|=1$, the set of all $z \in U$ for which

$$
|\arg (1-\bar{\tau} z)|<\alpha<\frac{\pi}{2},|z-\tau|<\varepsilon,
$$

where $\alpha$ is a given angle and $\varepsilon$ is so small that the boundary of the resulting set has only the point $\tau$ in common with the boundary of $U$, is called a symmetric Stolz angle with vertex $\tau$ and opening $2 \alpha$, and will be denoted by $A_{\tau, \alpha}$.

We now define what is meant by a set of $\rho$-discs for a complex-valued function $f$ defined in $U$ and taking its values in the $w$-plane [5]. We shall say that $f$ possesses $a$ set of $\rho$-discs if for any prescribed sequences $\left\{L_{\nu} \mid \nu=1,2, \ldots\right\}$ and $\left\{\left.\varepsilon_{\nu}\right|_{\nu}=1,2, \ldots\right\}$

where

(A) $1<L_{1}<L_{2}<\cdots<L_{\nu}<L_{\nu+1}<\cdots, \lim _{\nu \rightarrow \infty} L_{\nu}=\infty$, and

Received February 1, 1961. 
(B) $1>\varepsilon_{1}>\varepsilon_{2}>\cdots>\varepsilon_{\nu}>\varepsilon_{\nu+1}>\cdots, \lim _{\nu \rightarrow \infty} \varepsilon_{\nu}=0$,

there exists a sequence $\left\{D_{\nu} \mid \eta=1,2, \ldots\right\}$ of discs $D_{\nu} \equiv\left\{z \mid d\left(\zeta_{\nu}, z\right)<\varepsilon_{\nu}\right\}$ in $U$, having the following properties:

(C) $\left|\zeta_{\nu}\right|<\left|\zeta_{\nu+1}\right|, \nu=1,2, \ldots$;

(D) $\lim _{\nu \rightarrow \infty} d\left(D_{\llcorner}, D_{\nu+1}\right)=\infty$;

(E) in each $\operatorname{disc} D_{v}, \nu=1,2, \ldots$, the function $f$ assumes all values $w$ satisfying $|w|<L_{\nu}$, with the possible exception of a set of values, $E_{\nu}$, whose diameter is less than $\frac{2}{L_{\nu}}$.

Any such sequence $\left\{D_{\nu}\right\}$ or any infinite subsequence $\left\{D_{v_{k}} \mid k=1,2, \ldots\right\}$ of $\left\{D_{\nu}\right\}$ will then be called $a$ set of $\rho$-discs for $f$.

The set of centers $\left\{\zeta_{2}\right\}$ associated with such a set of discs is called $a$ set of $\rho$-points for $f$. In the set $\bigcup_{\nu=1}^{\infty} D_{\nu}$ the function $f$ obviously assumes infinitely often every finite complex value, with at most one exception. The term $\rho$ discs is used to indicate the analogy to Milloux's cercles de remplissage for entire functions. As pointed out in [5], it is possible to give other essentially equivalent definitions of a set of $\rho$-discs, and this has been done in a subsequent paper [1, p. 264], in which, with the aid of the concept of normal functions [6], generalizations of some of the results in [5] are established, in particular, results concerning what were called $\rho^{*}$-spirals in $[5, \mathrm{p} .271]$.

TheоRем I. Let $f$ be holomorphic in $U$ and let $\tau=e^{i / p}$ be a fixed point; $|\tau|=1$. Let $\left\{z_{n}\right\}$ and $\left\{z_{n}^{\prime}\right\}$ be sequences of points in $U$ having the following properties:

(i) $z_{n}=\rho_{n} e^{i, \rho} ; z_{n}^{\prime}=\rho_{n}^{\prime} e^{i, \rho} ; \rho_{n}>0, \rho_{n}^{\prime}>0$;

(ii) $\rho_{n}<\rho_{n}^{\prime}<\rho_{n+1}<\rho_{n+1}^{\prime}$;

(iii) $\lim _{n \rightarrow \infty} d\left(z_{n}, z_{n+1}\right)<\sigma, 0<\sigma<\infty$;

(iv) $f\left(z_{n}^{\prime}\right) \rightarrow \infty$ as $n \rightarrow \infty$;

(v) $\left|f\left(z_{n}\right)\right|<M$, where $M$ is some finite constant; $n=1,2, \ldots$ Then there exists a set of $\rho$-discs $\left\{D_{\nu}\right\}$ of $f$ such that $\bigcup_{\nu=1}^{\infty} D_{\nu} \subset \Delta_{\tau, \beta}$, where $\beta$ is. any angle which satisfies $0<\beta<\frac{\pi}{2}$ and $\sigma<\delta(\beta) \equiv \frac{1}{2} \log \cot \left(\frac{\pi}{4}-\frac{\beta}{2}\right)$.

Proof. To prove the theorem we employ the following lemma which is 
based on Schottky's theorem and is part of a lemma proved in [5, Lemma 1$]$.

LEMMA 1. Let $\eta$ and $\lambda$ be given numbers satisfying $0<\eta<\lambda<\infty$. Let $f$ be holomorphic in the disc $\left\{z \mid d\left(z_{0}, z\right)<\lambda\right\}$ and let it fail to assume the values 0 and 1 there. If $\left|f\left(z_{0}\right)\right|>E_{1}$, where $E_{1}$ is a certain positive constant which depends only on $\eta$ and $\lambda$, it then follows that for all $z$ in the disc $\left\{z \mid d\left(z_{0}, z\right)\right.$ $\leq \lambda-\eta\}$ we have $\left|f\left(z_{0}\right)\right|^{E_{2}}<|f(z)|$, where $E_{2}$ is another positive constant depending only on $\eta$ and $\lambda$.

We now use this lemma, with $\eta=\frac{\sigma}{2}$ and $\lambda=\sigma$, to prove:

Lemma 2. The points $z_{n}^{\prime}$ of Theorem I have the following property: there exists an index $N$ such that for infinitely many $n>N$ the function $f$ assumes the value 0 or the value 1 in the disc $C_{n}(\sigma) \equiv\left\{z \mid d\left(z_{n}^{\prime}, z\right)<\sigma\right\}$.

Proof. By conditions (i)-(iii) of Theorem I, we know that for infinitely many $n \geq$ some $n_{1}$ the disc $\bar{C}_{n}\left(\begin{array}{c}\sigma \\ 2\end{array}\right) \equiv\left\{z \mid d\left(z_{n}^{\prime}, z\right) \leq \frac{\sigma}{2}\right\}$ contains a member of the sequence $\left\{z_{n}\right\}$. By condition (iv) and Lemma 1 , there exists an $N \geq n_{1}$ such that $n>N$ implies $M<\left|f\left(z_{n}^{\prime}\right)\right|^{F_{2}}<|f(z)|$ for all $z \in \bar{C}_{n}\left(\begin{array}{c}\sigma \\ 2\end{array}\right)$, if the $\operatorname{disc} C_{n}(\sigma)$ contains no points at which $f$ assumes the values 0 or 1 . This contradicts $(v)$, and proves Lemma 2.

The establishment of Lemma 2 now enables us to use Theorem I in [5] to assert the existence of a set of $\rho$-points $\left\{\zeta_{\nu}\right\}$ for $f$ and that these points are located in our discs $C_{n}(\sigma)$. Now $\bigcup_{n=1}^{\infty} C_{n}(\sigma)$ is bounded by two hypercycles which are symmetric with respect to that diameter of the unit circle which terminates in $\tau$. At $\tau$ these hypercycles form angles $-\gamma$ and $\gamma$ with this diameter, $\gamma$ satisfying $0<\gamma<\frac{\pi}{2}, \sigma=\frac{1}{2} \log \cot \left(\frac{\pi}{4}-\frac{\gamma}{2}\right) \equiv \delta(\gamma)$. If we refer to condition (B) of the definition of $\rho$-discs, above, and consider appropriate hypercycles, we can readily assure ourselves that the $\rho$-discs associated with the $\zeta_{\nu}$ may be chosen to lie in $\Delta_{-, \beta}$, if $\beta$ satisfies $0<\beta<\frac{\pi}{2}$ and $\sigma<\delta(\beta)$. Theorem I is now proved.

Before proving the next theorem it is necessary to give several definitions. By a spiral we mean a simple continuous curve in $U$ given by $z=\zeta(t), 0 \leq t<\infty$, where $\lim _{t \rightarrow \infty}|\zeta(t)|=1$ and $\lim _{t \rightarrow \infty} \arg \zeta(t)=\infty$. If a spiral $S$ is given by $z=\zeta(t)$, then, for a given value of $t$ there exists a first value of $t$, say $t^{\prime}$, satisfying $t<t^{\prime}$ and $\arg \zeta\left(t^{\prime}\right)=\arg \zeta(t)+2 \pi$. We let $\bar{\mu}=\bar{\mu}(S) \equiv \lim _{t \rightarrow \infty} d\left(\zeta(t), \zeta\left(t^{\prime}\right)\right)$, this number giving us a measure of the "tightness" of the spiral $S$. By the symbol 
$(V, \bar{\mu}<\infty)$ we shall mean, as in [5], the family of all unbounded holomorphic functions in $U$ for each of which there exists some spiral $S$, with $\bar{\mu}(S)<\infty$, on which the function remains bounded.

The next theorem concerns spirals and Stolz angles.

Theorem II. If $f \in(V, \bar{\mu}<\infty)$, then for arbitrary $\tau$, with $|\tau|=1$, there exists $a$ set of $\rho$-discs for $f$ in $\dot{\Delta}_{\tau, \beta}^{*}$ if $\beta$ satisfies $0<\beta<\frac{\pi}{2}$ and $\bar{\mu}<\delta(\beta)$.

Proof. Let $S$ be the spiral in our hypothesis; that is, $|f(z)|<M$ for $z \in S$ and $\bar{\mu}(S)<\infty$. According to a theorem stated by Valiron [10], a theorem for which a correct proof is indicated in [1, Lemma 1], there exists a spiral $S^{\prime}$ along which $f$ tends to infinity. Letting $\tau=e^{i_{\rho}}$ be an arbitrary point on the boundary of $U$, letting $\varepsilon>0$ be given, referring to the definition of $\bar{\mu}$ and the fact that $S^{\prime}$ is a continuous curve, we can readily establish the existence of a pair of sequences $\left\{z_{n}\right\}$ and $\left\{z_{n}^{\prime}\right\}$, with $z_{n} \in S$ and $z_{n}^{\prime} \in S^{\prime}$, meeting all the requirements of the hypothesis of Theorem I for $\sigma=\bar{\mu}+\varepsilon$. The conclusion of Theorem II then follows from Theorem I.

Remark 1. If one established the inequality $\bar{\mu}\left(S^{\prime}\right) \leq 2 \bar{\mu}(S)$ and then used Theorem VIII of [5] to get an estimate on the size of the Stolz angle we are dealing with here, the resulting estimate would be weaker than the one given in Theorem II.

Remark 2. The work above, based on Schottky's theorem, yields new proofs of two results of Seidel [9, Theorem 2 and Corollary 1], results there proved with the aid of normal family arguments. See Remark 3, below.

The next theorem puts into a more general setting the basic argument employed in the theorems above.

THEOREM III.T Let $f$ be holomorphic in $U$ and let $A$ be any set $A \subset U$, such that $\bar{A} \cap(\bar{U}-U) \neq \varnothing$. If there exist sequences $\left\{z_{n}\right\}$ and $\left\{z_{n}^{\prime}\right\}$ in $A$ such that

(i) $\lim _{n \rightarrow \infty} d\left(z_{n}, z_{n}^{\prime}\right)=\sigma^{\prime}<\sigma, 0<\sigma<\infty$;

(ii) $\lim _{n \rightarrow \infty} f\left(z_{n}^{\prime}\right)=\infty$;

(iii) $\left|f\left(z_{n}\right)\right|<M$, where $M$ is a finite constant; $n=1,2, \ldots$; then there exists $a$ set of $\rho$-points for $f$ in the set $A^{(\sigma)} \equiv\{z \mid d(A, z)<\sigma\} . \quad(d(A, z)$ $\left.=\operatorname{g.l.b.}\left\{d\left(z^{\prime}, z\right) \mid z^{\prime} \in A\right\}\right)$. 
Proof. Using Theorem I in [5], all we must do here is to show that for all sufficiently large $n$ the discs $C_{n}(\sigma) \equiv\left\{z \mid d\left(z_{n}^{\prime}, z\right)<\sigma\right\}$ each contain a point $\zeta_{n}$ such that either $f\left(\zeta_{n}\right)=0$ or $f\left(\zeta_{n}\right)=1$. This we easily accomplish with the aid, once more, of Lemma 1 , above. For if $n$ is large enough, the conditions in the hypothesis of Theorem III insure both the existence of a point $z_{n} \in \bar{C}_{n}\left(\frac{\sigma^{\prime}+\sigma}{2}\right)$ and the validity of the inequality

$$
M<\left|f\left(z_{n}^{\prime}\right)\right|^{E_{2}}<|f(z)|
$$

for all $z \in \bar{C}_{n}\left(\frac{\sigma^{\prime}+\sigma}{2}\right) \equiv\left\{z \mid d\left(z_{n}^{\prime}, z\right) \leq \frac{\sigma^{\prime}+\sigma}{2}\right\}$, if $C_{n}(\sigma)$ does not contain points $\zeta$ at which $f$ assumes the value 0 or 1 . This would contradict condition (iii) of our hypothesis and hence Theorem III is proved.

To state our next theorem we need the following definition $[3, \mathrm{p} .121]$. If $A$ is any subset of $U$ such that $\bar{A} \cap(\vec{U}-U) \neq \varnothing$ then by the symbol $R(f, A)$ we mean the set of all finite complex values $a$ for each of which there exists a sequence of points $\left\{z_{n}\right\}$, with $z_{n} \in A$, and $\lim _{n \rightarrow \infty}\left|z_{n}\right|=1$, such that $f\left(z_{n}\right)=a$. By $\mathbf{C} R(f, A)$ we mean the complement of $R(f, A)$ with respect to the set of all finite complex numbers.

Theorem IV. Let $f$ be holomorphic in $U$ and let $A$ be any set $A \subset U$, where $\bar{A} \cap(\vec{U}-U) \neq \varnothing$. Let $\mathbf{C} R(f, \mathrm{~A})$ consist of at most one (finite) complex value. If there exist a positive constant $\sigma$ and a sequence $\left\{z_{n}^{\prime}\right\}$ of points $z_{n}^{\prime} \in A$ such that

(i) $\lim _{n \rightarrow \infty} f\left(z_{n}^{\prime}\right)=\infty$, and

(ii)

$$
\bigcup_{n=1}^{\infty}\left\{z \mid d\left(z_{n}^{\prime}, z\right)<\sigma\right\} \supset A \text {, }
$$

then there exists a set of $\rho$-points for $f$ lying in the set

$$
A^{(\sigma)} \equiv\{z \mid d(A, z)<\sigma\} .
$$

Proof. Here again we use Theorem I in [5], together with an argument similar to the one used in proving Theorem VII in [5]. Since $\mathbf{C} R(f, A)$ consists of at most one finite complex value, there exists a sequence $\left\{\zeta_{k}^{\prime}\right\}$ of points $\zeta_{k}^{\prime} \in A$ such that for each $k$ either $f\left(\zeta_{k}^{\prime}\right)=0$ or $f\left(\zeta_{k}^{\prime}\right)=1$. We are then assured, by conditions (i) and (ii) in our present hypothesis, of the existence of a sequence of $\operatorname{discs} C_{n}(\sigma) \equiv\left\{z \mid d\left(z_{n}^{\prime}, z\right)<\sigma\right\}$ in each of which the function $f$ 
assumes the values 0 or 1 and such that $\lim _{n \rightarrow \infty} f\left(z_{n}^{\prime}\right)=\infty$. By Theorem I in [5] there exists a set of $\rho$-points $\left\{\zeta_{\nu}\right\}$ of $f$ associated with the set $A$. These $\rho$ points need not all lie in $A$, (see condition 3 of Theorem I in [5] and the proof of Theorem VII in [5], but we can say that they necessarily satisfy $d\left(A, \zeta_{v}\right)<\sigma$ for every $\nu$. Our theorem is proved.

Remark 3. The nature of the set $A$ in special cases may, of course, permit us to assert the existence of a set of $\rho$-points lying in a set which is smaller than $A^{(\sigma)}$. To illustrate this, we consider the theorem of Seidel mentioned in Remark 2 , above. In our notation, his theorem reads :

If $f \in(V, \bar{\mu}<\infty)$, then for arbitrary $\tau$, where $|\tau|=1$, the set $\mathbf{C} R\left(f, \Delta_{\tau, x}\right)$ consists of at most one finite complex value for every $\Delta_{\tau, \alpha}$ for which $\bar{\mu}<\delta(\alpha)$.

If we now applied our Theorem IV (its hypothesis can be satisfied, with $\sigma=\mu$, if we use the "Valiron spiral" occurring in our proof of Theorem II, above, and make repeated use of the Heine-Borel theorem), we could then say that there exists a set of $\rho$-points for $f$ lying in $\Delta_{\mathfrak{\tau}, \alpha}^{(\bar{\mu})}$. (If it happens that $\bar{\mu}=0$, we may let $\sigma$ be an arbitrary positive number and make the appropriate adjustment.) This latter estimate is not, however, as good as the one proved in Theorem II, above.

Since the primary use of Theorems III and IV may involve sets $A$ which are Stolz angles, it is perhaps worthwhile to record the following corollary concerning symmetric Stolz angles.

Corollary 1. If the set $A$ in Theorems III and IV is a (symmetric) Stolz angle $\Delta_{\tau, \alpha}$ then the set $\Delta_{\because, \alpha}^{(g)}$ may be replaced by any Stolz angle $\Delta_{\tau, \beta}$ satisfying $\delta(\beta)>\delta(\alpha)+\sigma$.

The proof of this corollary is easily effected by a consideration of hypercycles terminating in $\tau$. (See the argument which precedes Theorem VIII in [5].)

In [5] and [9] several non-Euclidean analogues to the classical lines of Julia for entire functions were established. (See the $d_{0}$-spiral of Seidel in $[9, \mathrm{p}$. 162]; [5, p. 273]; the $\rho$-spiral, the $\rho^{*}$-spiral and the $\rho$-line in $[5$, p. 271 and p. $276]$. See also $[1$, p. 264]). We state below an elementary corollary involving the $\rho$-line. 
If $A$ is a radial line joining the center 0 of the unit circle to a point $\tau,|\tau|=1$, such that in the angular sector determined by an arbitrarily prescribed positive angle $\alpha$ having its vertex at 0 and having $d$ as its bisector there is to be found a set of $\rho$-discs for a given function $f$, the line $A$ is called $a \rho$-line of $f$.

The proof of this corollary is then clear:

Corollary 2. If $f$ and A satisfy the hypothesis of Theorem III (or IV), there exists a p-line of $f$ terminating in $\bar{A}^{(\sigma)} \cap(\bar{U}-U)=\bar{A} \cap(\bar{U}-U)$.

Of course, this $\rho$-line terminates in $\tau$ if $A$ is a Stol $z$ angle at $\tau$.

\section{REFERENCES}

[1] F. Bagemihl and W. Seidel, Behavior of meromorphic functions on boundary paths, with applications to normal functions. Archiv der Mathematik, 12 (1960), 263-269.

[2] C. Carathéodory, Conformal representation, Cambridge, 1952.

[ 3 ] E. F. Collingwood and M. L. Cartwright, Boundary theorems for a function meromorphic in the unit circle. Acta Math. 87 (1952), 83-146.

[4] L. H. Lange, A non-Euclidean analogue to a theorem of H. Milloux and its relationship to a theorem of W. Seidel. Amer. Math. Soc. Notices 5 (1958), 847-848.

[ 5 ] L. H. Lange, Sur les cercles de remplissage non-euclidiens. Ann. Sci. Ecol. Norm. Sup. (3), 77 (1960), 257-280.

[6] O. Lehto and K. I. Virtanen, Boundary behavior of normal meromorphic functions. Acta Math. 97 (1957), 47-65.

[7] H. Milloux, Le théorème de M. Picard. Suites de fonctions holomorphes. Fonctions móromorphes et fonctions entières. J. Math. pures et appl. (9), 3 (1924), 345-401.

[ 8] H. Milloux, Sur le théorème de M. Picard. Bull. Soc. Math. Fr. 53 (1925), 181-207.

[ 9 ] W. Seidel, Holomorphic functions with spiral asymptotic paths. Nagoya Math J. 14 (1959), 159-171.

[10] G. Valiron, Sur les singularités de certaines fonctions holomorphes et de leurs inverses. J. Math. pures et appl. (9), 15 (1936), 423-435.

\section{San Jose State College}

